\title{
The existence of solutions for nonlinear fractional multipoint boundary value problems at resonance
}

\author{
Na Xu, Wenbin Liu* and Lishun Xiao
}

${ }^{*}$ Correspondence: wblium@163.com

College of Sciences, China University of Mining and

Technology, Xuzhou, Jiangsu, 221116, P.R. China

\begin{abstract}
A class of nonlinear fractional multipoint boundary value problems at resonance is considered in this article. The existence results are obtained by the method of the coincidence degree theory of Mawhin. An example is given to illustrate the results. MSC: $34 \mathrm{~A} 08$

Keywords: coincidence degree; fractional differential equation; resonance; multipoint boundary conditions
\end{abstract}

\section{Introduction}

The subject of fractional calculus has gained considerable popularity during the past decades, due mainly to its frequent appearance in a variety of different areas such as physics, aerodynamics, polymer rheology, etc. (see [1-3]). Many methods have been introduced for solving fractional differential equations (FDEs for short in the remaining), such as the Laplace transform method, the iteration method, the Fourier transform method, etc. (see [4]).

Recently, there have been many works related to the existence of solutions for multipoint boundary value problems (BVPs for short in the remaining) at nonresonance of FDEs (see [5-11]). Motivated by the above articles and recent studies on FDEs (see [12-19]), we consider the existence of solutions for a nonlinear fractional multipoint BVPs at resonance in this article.

In [16], Zhang and Bai considered the following fractional three-point boundary value problems at resonance:

$$
\left\{\begin{array}{l}
D_{0+}^{\alpha} u(t)=f\left(t, u(t), D_{0+}^{\alpha-(n-1)} u(t), \ldots, D_{0+}^{\alpha-1} u(t)\right)+e(t), \quad 0<t<1, \\
I_{0+}^{n-\alpha} u(0)=D_{0+}^{\alpha-(n-1)} u(0)=\cdots=D_{0+}^{\alpha-2} u(0)=0, \quad u(1)=\sigma u(\eta),
\end{array}\right.
$$

where $n>2$ is a natural number; $n-1<\alpha \leq n$ is a real number; $D_{0+}^{\alpha}$ and $I_{0+}^{\alpha}$ are the standard Riemann-Liouville derivative and integral respectively; $f:[0,1] \times \mathbf{R}^{n} \mapsto \mathbf{R}$ is continuous; $e(t) \in L[0,1] ; \sigma \in(0,+\infty), \eta \in(0,1)$ are given constants such that $\sigma \eta^{\alpha-1}=1$. In their article, they made the operator $L u=D_{0_{+}}^{\alpha} u$ and got $\operatorname{dim} \operatorname{Ker} L=1$. In [17], Bai discussed fractional $m$-point boundary value problems at resonance with the case of $\operatorname{dim} \operatorname{Ker} L=1$.

In 2010, Bai and Jiang studied the fractional differential equation of boundary value problems at resonance with the case of $\operatorname{dim} \operatorname{Ker} L=2$ respectively (see [18, 19]), and we 
can see that they obtained the results by the assumption that a specific algebraic expression is not equal to zero; for example,

$$
R=\frac{1}{\alpha} \eta^{\alpha} \frac{\Gamma(\alpha) \Gamma(\alpha-1)}{\Gamma(2 \alpha-1)}\left[1-\sum_{i=1}^{m} \alpha_{i} \eta_{i}^{2 \alpha-2}\right]-\frac{1}{\alpha-1} \eta^{\alpha-1} \frac{\Gamma^{2}(\alpha)}{\Gamma(2 \alpha)}\left[1-\sum_{i=1}^{m} \alpha_{i} \eta_{i}^{2 \alpha-1}\right] \neq 0
$$

is referred to as a condition in [18]. We will show that the assumption like above $R \neq 0$ is not necessary.

In this article, we will use the coincidence degree theory to study the existence of solutions for a nonlinear FDEs at resonance which is given by

$$
D_{0+}^{\alpha} u(t)=f\left(t, u(t), D_{0+}^{\alpha-1} u(t), D_{0+}^{\alpha-2} u(t)\right), \quad 0<t<1,
$$

with boundary conditions

$$
\begin{aligned}
& \left.I_{0+}^{3-\alpha} u(t)\right|_{t=0}=0, \quad D_{0+}^{\alpha-1} u(1)=\sum_{i=1}^{m} a_{i} D_{0+}^{\alpha-1} u\left(\xi_{i}\right), \\
& D_{0+}^{\alpha-2} u(1)=\sum_{i=1}^{m} b_{i} D_{0+}^{\alpha-2} u\left(\eta_{i}\right),
\end{aligned}
$$

where $2<\alpha \leq 3 ; 0<\xi_{1}<\cdots<\xi_{m}<1 ; 0<\eta_{1}<\cdots<\eta_{m}<1 ; a_{i}, b_{i} \in \mathbf{R} ; f:[0,1] \times \mathbf{R}^{3} \mapsto \mathbf{R}$ with satisfying Carathéodory conditions; $D_{0^{+}}^{\alpha}$ and $I_{0^{+}}^{\alpha}$ are the standard Riemann-Liouville fractional derivative and fractional integral respectively.

BVPs (1.1)-(1.2) being at resonance means that the associated linear homogeneous equation $D_{0+}^{\alpha} u(t)=0$ with boundary conditions (1.2) has $u(t)=a t^{\alpha-1}+b t^{\alpha-2}$ as a nontrivial solution, where $0<t<1, a, b \in \mathbf{R}$.

We will always suppose that the following conditions hold:

$$
\sum_{i=1}^{m} a_{i}=1, \quad \sum_{i=1}^{m} b_{i} \eta_{i}=1, \quad \sum_{i=1}^{m} b_{i}=1
$$

The rest of this article is organized as follows: In Section 2, we give some definitions, lemmas and notations. In Section 3, we establish theorems of existence result for BVPs (1.1)-(1.2). In Section 4, we give an example to illustrate our result.

\section{Preliminaries}

We present here some necessary basic knowledge and definitions of the fractional calculus theory, which can be found in [1-3].

Definition 2.1 The Riemann-Liouville fractional integral of order $\alpha>0$ of a function $y$ : $(0, \infty) \mapsto \mathbf{R}$ is given by

$$
I_{0+}^{\alpha} y(t)=\frac{1}{\Gamma(\alpha)} \int_{0}^{t}(t-s)^{\alpha-1} y(s) \mathrm{d} s
$$

where $\Gamma(\cdot)$ is the Gamma function, provided the right side is pointwise defined on $(0, \infty)$. 
Definition 2.2 The Riemann-Liouville fractional derivative of order $\alpha>0$ of a function $y:(0, \infty) \mapsto \mathbf{R}$ is given by

$$
D_{0+}^{\alpha} y(t)=\frac{1}{\Gamma(n-\alpha)}\left(\frac{\mathrm{d}}{\mathrm{d} t}\right)^{n} \int_{0}^{t} \frac{y(s)}{(t-s)^{\alpha-n+1}} \mathrm{~d} s
$$

where $n=[\alpha]+1$, provided the right side is pointwise defined on $(0, \infty)$.

Definition 2.3 ([18]) We say that the $\operatorname{map} f:[0,1] \times \mathbf{R}^{n} \mapsto \mathbf{R}$ satisfies Carathéodory conditions with respect to $L[0,1]$ if the following conditions are satisfied:

(i) for each $z \in \mathbf{R}^{n}$, the mapping $t \mapsto f(t, z)$ is Lebesgue measurable;

(ii) for almost every $t \in[0,1]$, the mapping $t \mapsto f(t, z)$ is continuous on $\mathbf{R}^{n}$;

(iii) for each $E>0$, there exists a $\rho_{E} \in L[0,1]$ such that, for a.e. $t \in[0,1]$ and every $|u| \leq E$, we have $f(t, u) \leq \rho_{E}(t)$.

Lemma 2.4 ([2]) Assume $y(t) \in C[0,1] \cap L[0,1], 0 \leq \beta \leq \alpha$, then $D_{0^{+}}^{\beta} I_{0^{+}}^{\alpha} y(t)=I_{0^{+}}^{\alpha-\beta} y(t)$. And, for all $\alpha \geq 0, \beta>-1$, we have that

$$
I_{0^{+}}^{\alpha} t^{\beta}=\frac{\Gamma(\beta+1)}{\Gamma(\alpha+\beta+1)} t^{\alpha+\beta}, \quad D_{0^{+}}^{\alpha} t^{\beta}=\frac{\Gamma(\beta+1)}{\Gamma(\beta-\alpha+1)} t^{\beta-\alpha} .
$$

Lemma 2.5 ([2]) Let $\alpha>0, n=[\alpha]+1$ and assume that $y, D_{0^{+}}^{\alpha} y \in L(0,1)$, then the following equality holds almost everywhere on $[0,1]$,

$$
\left(I_{0+}^{\alpha} D_{0+}^{\alpha} y\right)(t)=y(t)-\sum_{i=1}^{n} \frac{\left.\left(\left(I_{0+}^{n-\alpha} y\right)(t)\right)^{n-i}\right|_{t=0}}{\Gamma(\alpha-i+1)} t^{\alpha-i}
$$

Now, we briefly recall some notations and an abstract existence result, which can be found in [20]. Let $Y, Z$ be real Banach spaces, $L: \operatorname{dom} L \subset Y \mapsto Z$ be a Fredholm map of index zero, and $P: Y \mapsto Y, Q: Z \mapsto Z$ be continuous projectors such that

$$
\operatorname{Im} P=\operatorname{Ker} L, \quad \operatorname{Ker} Q=\operatorname{Im} L, \quad Y=\operatorname{Ker} L \oplus \operatorname{Ker} P, \quad Z=\operatorname{Im} Q \oplus \operatorname{Im} L .
$$

It follows that $\left.L\right|_{\operatorname{dom} L \cap \operatorname{Ker} P}: \operatorname{dom} L \cap \operatorname{Ker} P \mapsto \operatorname{Im} L$ is invertible. We denote the inverse by $K_{p}$. If $\Omega$ is an open bounded subset of $Y$ such that $\operatorname{dom} L \cap \Omega \neq \varnothing$, the map $N: Y \mapsto Z$ will be called $L$-compact on $\Omega$ if $Q N(\bar{\Omega})$ is bounded and $K_{p}(I-Q) N: \bar{\Omega} \mapsto Y$ is compact.

Lemma 2.6 ([20]) Let L be a Fredholm operator of index zero and $N$ be L-compact on $\bar{\Omega}$. The equation $L x=N x$ has at least one solution in $\operatorname{dom} L \cap \bar{\Omega}$ if the following conditions are satisfied:

(i) $L x \neq \lambda N x$ for each $(x, \lambda) \in[\operatorname{dom} L \backslash \operatorname{Ker} L \cap \partial \Omega] \times[0,1]$;

(ii) $N x \notin \operatorname{Im} L$ for each $x \in \operatorname{Ker} L \cap \partial \Omega$;

(iii) $\operatorname{deg}\left(\left.J Q N\right|_{\operatorname{Ker} L}, \operatorname{Ker} L \cap \Omega, 0\right) \neq 0$,

where $Q: Z \mapsto Z$ is a projection such that $\operatorname{Ker} Q=\operatorname{Im} L$ and $J: \operatorname{Im} Q \mapsto \operatorname{Ker} L$ is a any isomorphism.

In this article, we use the Banach space $C[0,1]$ with the norm $\|u\|_{\infty}=\max _{t \in[0,1]}|u(t)|$. 
Lemma 2.7 ([16]) Given $\mu>0$ and $N=[\mu]+1$, for any $x \in C[0,1], c_{i} \in \mathbf{R}(i=1,2, \ldots, N-1)$, we can define a Banach space

$$
C^{\mu}[0,1]=\left\{u(t) \mid u(t)=I_{0^{+}}^{\mu} x(t)+c_{1} t^{\mu-1}+c_{2} t^{\mu-2}+\cdots+c_{N-1} t^{\mu-(N-1)}, t \in(0,1)\right\},
$$

with the norm defined by $\|u\|_{C^{\mu}}=\left\|D_{0^{+}}^{\mu} u\right\|_{\infty}+\cdots+\left\|D_{0^{+}}^{\mu-(N-1)} u\right\|_{\infty}+\|u\|_{\infty}$.

Lemma 2.8 ([16]) $E \subset C^{\mu}[0,1]$ is a sequentially compact set if and only if $E$ is uniformly bounded and equicontinuous. Here, a uniform bound means that there exists a constant $M>0$ with each $u \in E$, such that

$$
\|u\|_{C^{\mu}}=\left\|D_{0+}^{\mu} u\right\|_{\infty}+\cdots+\left\|D_{0+}^{\mu-(N-1)} u\right\|_{\infty}+\|u\|_{\infty}<M
$$

and equicontinuation means that there exists a $\delta>0$ with $\left|t_{1}-t_{2}\right|<\delta$ for any $t_{1}, t_{2} \in[0,1]$, $u \in E$ and $\varepsilon>0$, such that

$$
\left|u\left(t_{1}\right)-u\left(t_{2}\right)\right|<\varepsilon, \quad\left|D_{0+}^{\alpha-i} u\left(t_{1}\right)-D_{0+}^{\alpha-i} u\left(t_{2}\right)\right|<\varepsilon \quad(i=1,2, \ldots, N-1) .
$$

In this article, let $Z=L[0,1]$ with the norm $\|y\|_{1}=\int_{0}^{1}|y(s)| \mathrm{d} s$ and $Y=C^{\alpha-1}[0,1]$ with the norm $\|u\|_{Y}=\left\|D_{0^{+}}^{\alpha-1} u\right\|_{\infty}+\left\|D_{0^{+}}^{\alpha-2} u\right\|_{\infty}+\|u\|_{\infty}$. Define the operator $L: \operatorname{dom} L \cap Y \mapsto Z$ by

$$
L u=D_{0^{+}}^{\alpha} u,
$$

where $\operatorname{dom} L=\left\{u \in C^{\alpha-1}[0,1] \mid D_{0+}^{\alpha} u \in Z, u\right.$ satisfies (1.2) $\}$. Define the operator $N: Y \mapsto Z$ by

$$
N u(t)=f\left(t, u(t), D_{0+}^{\alpha-1} u(t), D_{0+}^{\alpha-2} u(t)\right), \quad \forall t \in[0,1] .
$$

Thus, BVP (1.1) can be written as $L u=N u$ for each $u \in \operatorname{dom} L$.

\section{Main results}

First, let us introduce the following notations for convenience, with setting $p \in\{1,2, \ldots, m-$ 1\} and $q \in \mathbf{Z}^{+}$with $q \geq p+1$,

$$
\begin{aligned}
& \Lambda_{1}:=\frac{1}{p(p+1)}\left(1-\sum_{i=1}^{m} b_{i} \eta_{i}^{p+1}\right), \quad \Lambda_{2}:=\frac{1}{q(q-1)}\left(1-\sum_{i=1}^{m} b_{i} \eta_{i}^{q}\right) \\
& \Lambda_{3}:=\frac{1}{p}\left(1-\sum_{i=1}^{m} a_{i} \xi_{i}^{p}\right), \quad \Lambda_{4}:=\frac{1}{q-1}\left(1-\sum_{i=1}^{m} a_{i} \xi_{i}^{q-1}\right) \\
& \Lambda:=\Lambda_{1} \Lambda_{4}-\Lambda_{2} \Lambda_{3}, \quad \mu:=3+\frac{1}{\Gamma(\alpha)}+\frac{1}{\Gamma(\alpha-1)} \\
& \omega:=2+\frac{1}{\Gamma(\alpha)}, \quad \rho:=5+\frac{2}{\Gamma(\alpha)}+\frac{1}{\Gamma(\alpha-1)} .
\end{aligned}
$$

Then, let us make some assumptions which will be used throughout the article. 
(H1) There exist functions $h(t), r(t), s(t), w(t), e(t) \in L[0,1]$ and a constant $\theta \in[0,1)$ such that for all $(x, y, z) \in \mathbf{R}^{3}, t \in[0,1]$,

$$
|f(t, x, y, z)| \leq h(t)|x|+r(t)|y|+s(t)|z|+w(t)|z|^{\theta}+e(t)
$$

(H2) For any $u \in \operatorname{dom} L, t \in[0,1]$, there exists a constant $A>0$ such that if $D_{0^{+}}^{\alpha-1} u(t)>A$, then either

$$
\Lambda_{2} T_{1} N u-\Lambda_{4} T_{2} N u<0, \quad \text { or } \quad \Lambda_{2} T_{1} N u-\Lambda_{4} T_{2} N u>0 ;
$$

(H3) For any $u \in \operatorname{dom} L, t \in[0,1]$, there exists a constant $B>0$ such that if $D_{0^{+}}^{\alpha-2} u(t)>B$, then either

$$
\Lambda_{1} T_{1} N u-\Lambda_{3} T_{2} N u>0, \quad \text { or } \quad \Lambda_{1} T_{1} N u-\Lambda_{3} T_{2} N u<0 .
$$

Theorem 3.1 If conditions (C), (H1)-(H3) hold, then BVPs (1.1)-(1.2) have at least one solution provided that $\rho\left(\|h\|_{1}+\|r\|_{1}+\|s\|_{1}\right)<1$.

In order to obtain our main result, we first present and prove Lemmas 3.2-3.8. Now, let us define operators $T_{j}: Z \mapsto Z(j=1,2)$ as follows:

$$
\begin{aligned}
& T_{1} x(t)=\int_{0}^{1} x(s) \mathrm{d} s-\sum_{i=1}^{m} a_{i} \int_{0}^{\xi_{i}} x(s) \mathrm{d} s, \quad \forall t \in[0,1] \\
& T_{2} x(t)=\int_{0}^{1}(1-s) x(s) \mathrm{d} s-\sum_{i=1}^{m} b_{i} \int_{0}^{\eta_{i}}\left(\eta_{i}-s\right) x(s) \mathrm{d} s, \quad \forall t \in[0,1] .
\end{aligned}
$$

Lemma 3.2 If condition (C) holds and L is defined by (2.1), then

$$
\operatorname{Ker} L=\left\{a t^{\alpha-1}+b t^{\alpha-2} \mid a, b \in \mathbf{R}\right\}, \quad \operatorname{Im} L=\left\{x \in Z \mid T_{j} x=0, j=1,2\right\} .
$$

Proof By (2.1) and Lemma 2.5, $D_{0+}^{\alpha} u(t)=0$ has a solution

$$
u(t)=\frac{\left.D_{0+}^{\alpha-1} u(t)\right|_{t=0}}{\Gamma(\alpha)} t^{\alpha-1}+\frac{\left.D_{0+}^{\alpha-2} u(t)\right|_{t=0}}{\Gamma(\alpha-1)} t^{\alpha-2}+\frac{\left.I_{0+}^{3-\alpha} u(t)\right|_{t=0}}{\Gamma(\alpha-2)} t^{\alpha-3} .
$$

Combining with the condition (1.2), we get $\operatorname{Ker} L=\left\{a t^{\alpha-1}+b t^{\alpha-2} \mid a, b \in \mathbf{R}\right\} \cong \mathbf{R}^{2}$.

Suppose $\forall x \in \operatorname{Im} L$, then there exists $u \in \operatorname{dom} L$ such that $x=L u$, i.e., $u \in Y, x=D_{0+}^{\alpha} u$. By Lemma 2.5, we have

$$
I_{0+}^{\alpha} x(t)=u(t)-\frac{\left.D_{0+}^{\alpha-1} u(t)\right|_{t=0}}{\Gamma(\alpha)} t^{\alpha-1}-\frac{\left.D_{0+}^{\alpha-2} u(t)\right|_{t=0}}{\Gamma(\alpha-1)} t^{\alpha-2}-\frac{\left.I_{0+}^{3-\alpha} u(t)\right|_{t=0}}{\Gamma(\alpha-2)} t^{\alpha-3} .
$$

Then in view of condition (C), (1.2) and Lemma 2.4, $x$ satisfies

$$
\left\{\begin{array}{l}
\int_{0}^{1} x(s) \mathrm{d} s-\sum_{i=1}^{m} a_{i} \int_{0}^{\xi_{i}} x(s) \mathrm{d} s=0 \\
\int_{0}^{1}(1-s) x(s) \mathrm{d} s-\sum_{i=1}^{m} b_{i} \int_{0}^{\eta_{i}}\left(\eta_{i}-s\right) x(s) \mathrm{d} s=0
\end{array}\right.
$$


On the other hand, suppose $\forall x \in Z$ and it satisfies (3.1), let $u(t)=I_{0+}^{\alpha} x(t)$, then $u \in \operatorname{dom} L$, $D_{0+}^{\alpha} u(t)=x(t)$, i.e., $x \in \operatorname{Im} L$. Therefore, we obtain that

$$
\operatorname{Im} L=\left\{x \in Z \mid T_{j} x=0, j=1,2\right\} .
$$

Lemma 3.3 If condition (C) holds, then there exist two constants $q \in \mathbf{Z}^{+}$and $p \in$ $\{1,2, \ldots, m-1\}$ with $q \geq p+1$ such that $\Lambda \neq 0$.

Proof From $\sum_{i=1}^{m} a_{i}=1$, we obtain that for any nonnegative integer $l$, there exists $k_{l}-1 \in$ $\{l m+1, \ldots,(l+1) m\}$ such that $\sum_{i=1}^{m} a_{i} \xi_{i}^{k_{l}-1} \neq 1$. If else, we obtain that $\sum_{i=1}^{m} a_{i} \xi_{i}^{k_{l}-1}=1, k_{l}-1=$ $0, l m+1, \ldots,(l+1) m$.

If $l=0$, we have

$$
\left(\begin{array}{cccc}
1 & 1 & \cdots & 1 \\
\xi_{1} & \xi_{2} & \cdots & \xi_{m} \\
\vdots & \ddots & \vdots & \\
\xi_{1}^{m} & \xi_{2}^{m} & \cdots & \xi_{m}^{m}
\end{array}\right)\left(\begin{array}{c}
a_{1} \\
a_{2} \\
\vdots \\
a_{m}
\end{array}\right)=\left(\begin{array}{c}
1 \\
1 \\
\vdots \\
1
\end{array}\right)
$$

It is equal to

$$
\left(\begin{array}{cccc}
1-\xi_{1} & 1-\xi_{2} & \cdots & 1-\xi_{m} \\
\xi_{1}\left(1-\xi_{1}\right) & \xi_{2}\left(1-\xi_{2}\right) & \cdots & \xi_{m}\left(1-\xi_{m}\right) \\
\vdots & \ddots & \vdots & \\
\xi_{1}^{m-1}\left(1-\xi_{1}\right) & \xi_{2}^{m-1}\left(1-\xi_{2}\right) & \cdots & \xi_{m}^{m-1}\left(1-\xi_{m}\right)
\end{array}\right)\left(\begin{array}{c}
a_{1} \\
a_{2} \\
\vdots \\
a_{m}
\end{array}\right)=\left(\begin{array}{c}
0 \\
0 \\
\vdots \\
0
\end{array}\right) .
$$

Since the determinant of coefficients is not equal to zero, we have that $a_{i}=0(i=$ $1,2, \ldots, m)$, which is a contradiction to condition $(C)$.

If $l \in \mathbf{Z}^{+}$, we get

$$
\left(\begin{array}{cccc}
1 & 1 & \cdots & 1 \\
\xi_{1}^{l m+1} & \xi_{2}^{l m+1} & \cdots & \xi_{m}^{l m+1} \\
\vdots & \ddots & \vdots & \\
\xi_{1}^{l m+m} & \xi_{2}^{l m+m} & \cdots & \xi_{m}^{l m+m}
\end{array}\right)\left(\begin{array}{c}
a_{1} \\
a_{2} \\
\vdots \\
a_{m}
\end{array}\right)=\left(\begin{array}{c}
1 \\
1 \\
\vdots \\
1
\end{array}\right)
$$

Similarly, we can deduce that the determinant of coefficients is not equal to zero, so we have that $a_{i}=0(i=1,2, \ldots, m)$, which is a contradiction to condition $(C)$. Thus, there exists $k_{l}-1 \in\{l m+1, \ldots,(l+1) m\}$ such that $\sum_{i=1}^{m} a_{i} \xi_{i}^{k_{l}-1} \neq 1$.

Similarly, from $\sum_{i=1}^{m} b_{i}=\sum_{i=1}^{m} b_{i} \eta_{i}=1$, we have that there exists a constant $p \in\{1,2, \ldots$, $m-1$ \} such that

$$
\sum_{i=1}^{m} b_{i} \eta_{i}^{p+1} \neq 1
$$

Let

$$
S=\left\{\left(k_{l}-1\right) \in \mathbf{Z}^{+} \mid \frac{(p+1)\left(1-\sum_{i=1}^{m} b_{i} \eta_{i}^{k_{l}}\right)\left(1-\sum_{i=1}^{m} a_{i} \xi_{i}^{p}\right)}{k_{l}\left(1-\sum_{i=1}^{m} a_{i} \xi_{i}^{k_{l}-1}\right)}=1-\sum_{i=1}^{m} b_{i} \eta_{i}^{p+1}\right\},
$$


we shall prove that $S$ is a finite set. If else, there exists a strict increasing sequence $\left\{k_{l n}\right\}_{n=1}^{\infty}$ such that

$$
\frac{(p+1)\left(1-\sum_{i=1}^{m} b_{i} \eta_{i}^{k_{l n}}\right)\left(1-\sum_{i=1}^{m} a_{i} \xi_{i}^{p}\right)}{k_{l n}\left(1-\sum_{i=1}^{m} a_{i} \xi_{i}^{k_{l n}-1}\right)}=1-\sum_{i=1}^{m} b_{i} \eta_{i}^{p+1} .
$$

Since $\sum_{i=1}^{m} b_{i} \eta_{i}^{p+1} \neq 1$, we have $\sum_{i=1}^{m} a_{i} \xi_{i}^{p} \neq 1$. Thus,

$$
1-\sum_{i=1}^{m} b_{i} \eta_{i}^{p+1}=\lim _{k_{l n} \rightarrow+\infty} \frac{(p+1)\left(1-\sum_{i=1}^{m} b_{i} \eta_{i}^{k_{l n}}\right)\left(1-\sum_{i=1}^{m} a_{i} \xi_{i}^{p}\right)}{k_{l n}\left(1-\sum_{i=1}^{m} a_{i} \xi_{i}^{k_{l n}-1}\right)}=0
$$

which is a contradiction to (3.2). Therefore, there exists two constants $p \in\{1,2, \ldots, m-1\}$ and $q \in \mathbf{Z}^{+}$with $q \geq p+1$ such that $\Lambda \neq 0$.

Lemma 3.4 If the condition (C) holds and $L$ is defined by (2.1), then $L$ is a Fredholm operator of index zero. Define the linear operator $K_{p}: \operatorname{Im} L \mapsto \operatorname{dom} L \cap \operatorname{Ker} P$ with $K_{P} x=I_{0^{\alpha}}^{\alpha} x$, then it is the inverse of L. Furthermore, we have

$$
\left\|K_{p} x\right\|_{Y} \leq \omega\|x\|_{1}
$$

Proof For each $p \in\{1,2, \ldots, m-1\}$ and $q \in \mathbf{Z}^{+}$with $q \geq p+1$, define operator $Q: Z \mapsto Z$ by

$$
Q x(t)=\left(Q_{1} x(t)\right) t^{p-1}+\left(Q_{2} x(t)\right) t^{q-2}, \quad \forall t \in[0,1],
$$

where

$$
Q_{1} x(t)=\frac{1}{\Lambda}\left[-\Lambda_{2} T_{1} x(t)+\Lambda_{4} T_{2} x(t)\right], \quad Q_{2} x(t)=\frac{1}{\Lambda}\left[\Lambda_{1} T_{1} x(t)-\Lambda_{3} T_{2} x(t)\right] .
$$

It is clear that $\operatorname{dim} \operatorname{Im} Q_{1}=2$. It follows from (3.4), the definition of $T_{1}$ and $T_{2}$ that

$$
\begin{aligned}
Q_{1}\left(\left(Q_{1} x\right) t^{p-1}\right) & =\frac{1}{\Lambda}\left[-\Lambda_{2} T_{1}\left(\left(Q_{1} x\right) t^{p-1}\right)+\Lambda_{4} T_{2}\left(\left(Q_{1} x\right) t^{p-1}\right)\right] \\
& =\frac{1}{\Lambda}\left[-\Lambda_{2} \Lambda_{3}\left(Q_{1} x\right)+\Lambda_{1} \Lambda_{4}\left(Q_{1} x\right)\right]=Q_{1} x
\end{aligned}
$$

similarly, we can derive that

$$
Q_{1}\left(\left(Q_{2} x\right) t^{q-2}\right)=0, \quad Q_{2}\left(\left(Q_{1} x\right) t^{p-1}\right)=0, \quad Q_{2}\left(\left(Q_{2} x\right) t^{q-2}\right)=Q_{2} x .
$$

Hence, for each $x \in Z$ and $t \in[0,1]$, it follows from the (3.3)-(3.6) that

$$
\begin{aligned}
Q^{2} x & =Q_{1}\left[\left(Q_{1} x\right) t^{p-1}+\left(Q_{2} x\right) t^{q-2}\right] t^{p-1}+Q_{2}\left[\left(Q_{1} x\right) t^{p-1}+\left(Q_{2} x\right) t^{q-2}\right] t^{q-2} \\
& =\left(Q_{1} x\right) t^{p-1}+\left(Q_{2} x\right) t^{q-2}=Q x .
\end{aligned}
$$

Furthermore, $Q$ is a continuous linear projector. 
For each $x \in \operatorname{Im} L$, we have $Q x=0$, i.e., $x \in \operatorname{Ker} Q$. On the other hand, for each $x \in \operatorname{Ker} Q$, we have that

$$
\left\{\begin{array}{l}
-\Lambda_{2} T_{1} x+\Lambda_{4} T_{2} x=0 \\
\Lambda_{1} T_{1} x-\Lambda_{3} T_{2} x=0
\end{array}\right.
$$

However, the determinant of coefficients is as follows

$$
\left|\begin{array}{cc}
-\Lambda_{2} & \Lambda_{4} \\
\Lambda_{1} & -\Lambda_{3}
\end{array}\right|=-\Lambda \neq 0
$$

then we have $T_{j} x=0(j=1,2)$, i.e., $x \in \operatorname{Im} L$. Thus, $\operatorname{Ker} Q=\operatorname{Im} L$.

Take any $x \in Z$ in the type $x=(x-Q x)+Q x$, obviously, $x-Q x \in \operatorname{Ker} Q=\operatorname{Im} L$ and $Q x \in$ $\operatorname{Im} Q$, so $Z=\operatorname{Im} L+\operatorname{Im} Q$. For any $x \in \operatorname{Im} L \cap \operatorname{Im} Q$ with $x=a t^{p-1}+b t^{q-2}$, by Lemma 3.2, we have

$$
\begin{aligned}
& \int_{0}^{1}\left(a s^{p-1}+b s^{q-2}\right) \mathrm{d} s-\sum_{i=1}^{m} a_{i} \int_{0}^{\xi_{i}}\left(a s^{p-1}+b s^{q-2}\right) \mathrm{d} s=0, \\
& \int_{0}^{1}(1-s)\left(a s^{p-1}+b s^{q-2}\right) \mathrm{d} s-\sum_{i=1}^{m} b_{i} \int_{0}^{\eta_{i}}\left(\eta_{i}-s\right)\left(a s^{p-1}+b s^{q-2}\right) \mathrm{d} s=0 .
\end{aligned}
$$

That is,

$$
\left\{\begin{array}{l}
a \Lambda_{3}+b \Lambda_{4}=0 \\
a \Lambda_{1}+b \Lambda_{2}=0
\end{array}\right.
$$

but the determinant of coefficients is as follows

$$
\left|\begin{array}{cc}
\Lambda_{3} & \Lambda_{4} \\
\Lambda_{1} & \Lambda_{2}
\end{array}\right|=-\Lambda \neq 0,
$$

we can deduce that $a=b=0$. Hence, $\operatorname{Im} L \cap \operatorname{Im} Q=0$. Furthermore, we get $Z=\operatorname{Im} L \oplus$ $\operatorname{Im} Q$. Therefore, $\operatorname{dim} \operatorname{Ker} L=\operatorname{dim} \operatorname{Im} Q=\operatorname{codim} \operatorname{Im} L=2$, which means that $L$ is a Fredholm operator of index zero.

Let operator $P: Y \mapsto Y$ and

$$
P u(t)=\frac{\left.D_{0+}^{\alpha-1} u(t)\right|_{t=0}}{\Gamma(\alpha)} t^{\alpha-1}+\frac{\left.D_{0+}^{\alpha-2} u(t)\right|_{t=0}}{\Gamma(\alpha-1)} t^{\alpha-2}, \quad \forall t \in[0,1] .
$$

It is easy to calculate that $P u(t)=P^{2} u(t)$; furthermore, $P$ is a continuous linear projector. Obviously

$$
\operatorname{Ker} P=\left\{u \in Y \mid D_{0+}^{\alpha-1} u(0)=D_{0+}^{\alpha-2} u(0)=0\right\} .
$$

It is clear that $Y=\operatorname{Ker} L \oplus \operatorname{Ker} P$.

For any $x \in \operatorname{Im} L$, in view of the definition of operators $K p$ and $L$, we have $L K_{P} x=L I_{0_{+}}^{\alpha} x=$ $D_{0+}^{\alpha} I_{0+}^{\alpha} x=x$. On the other hand, if $u \in \operatorname{dom} L \cap \operatorname{Ker} P$, we have $D_{0+}^{\alpha-1} u(0)=D_{0+}^{\alpha-2} u(0)=0$, 
$u \in \operatorname{dom} L$. Therefore, by Lemma 2.5 and definitions of operators $K_{p}$ and $L$, we know that $\left(K_{p} L\right) u=u$, which implies that $K_{p}=\left[\left.L\right|_{\operatorname{dom} L \cap K e r P}\right]^{-1}$. By the definition of $K_{p}$, we have

$$
K_{p} x(t)=I_{0+}^{\alpha} x(t)=\frac{1}{\Gamma(\alpha)} \int_{0}^{t}(t-s)^{\alpha-1} x(s) \mathrm{d} s, \quad \forall x \in \operatorname{Im} L .
$$

It follows from Lemma 2.4 that

$$
D_{0+}^{\alpha-1}\left(K_{p} x\right)(t)=\int_{0}^{t}(t-s) x(s) \mathrm{d} s, \quad D_{0+}^{\alpha-2}\left(K_{p} x\right)(t)=\int_{0}^{t} x(s) \mathrm{d} s .
$$

Then, we have

$$
\left\|K_{p} x\right\|_{\infty} \leq \frac{1}{\Gamma(\alpha)}\|x\|_{1}, \quad\left\|D_{0+}^{\alpha-1}\left(K_{p} x\right)\right\|_{\infty} \leq\|x\|_{1}, \quad\left\|D_{0+}^{\alpha-2}\left(K_{p} x\right)\right\|_{\infty} \leq\|x\|_{1} .
$$

By the definition of the norm in space $Y$, we get $\left\|K_{p} x\right\|_{Y} \leq \omega\|x\|_{1}$.

Lemma 3.5 Assume $\Omega \subset Y$ is an open bounded subset such that $\operatorname{dom} L \cap \bar{\Omega} \neq \varnothing$, and $N$ is defined by (2.2), then $N$ is L-compact on $\bar{\Omega}$.

Proof In order to prove $N$ is $L$-compact, we only need to prove that $Q N(\bar{\Omega})$ is bounded and $K_{p}(I-Q) N(u): \bar{\Omega} \mapsto Y$ is compact. Since the function $f$ satisfies Carathéodory conditions and $u \in \bar{\Omega}$, for each $E>0$, there exists a $\rho_{E}(t) \in L[0,1]$ such that, for a.e. $t \in[0,1]$ and every $|u| \leq E$, we have $f \leq \rho_{E}$. By the definition of operators $Q$ and $K_{p}$ on the interval $[0,1]$, it is easy to get that $Q N(\bar{\Omega})$ and $K_{p}(I-Q) N(\bar{\Omega})$ are bounded. Thus, there exists a constant $r>0$ with each $t \in[0,1]$, such that $|Q N u(t)| \leq r$.

For all $0 \leq t_{1}<t_{2} \leq 1,2<\alpha \leq 3, u \in \bar{\Omega}$, we have

$$
\begin{aligned}
\left|K_{p}(I-Q) N u\left(t_{2}\right)-K_{p}(I-Q) N u\left(t_{1}\right)\right| \\
=\frac{1}{\Gamma(\alpha)} \mid \int_{0}^{t_{1}}\left[\left(t_{2}-s\right)^{\alpha-1}-\left(t_{1}-s\right)^{\alpha-1}\right](I-Q) N u(s) \mathrm{d} s \\
\quad+\int_{t_{1}}^{t_{2}}\left(t_{2}-s\right)^{\alpha-1}(I-Q) N u(s) \mathrm{d} s \mid \\
\leq \frac{1}{\Gamma(\alpha)}\left\{\int_{0}^{t_{1}}\left[\left(t_{2}-s\right)^{\alpha-1}-\left(t_{1}-s\right)^{\alpha-1}\right]\left(r+\left|\rho_{E}(s)\right|\right) \mathrm{d} s\right. \\
\left.\quad+\int_{t_{1}}^{t_{2}}\left(t_{2}-s\right)^{\alpha-1}\left(r+\left|\rho_{E}(s)\right|\right) \mathrm{d} s\right\} \\
\leq \frac{1}{\Gamma(\alpha)}\left\{\int_{0}^{t_{2}}\left|\rho_{E}(s)\right|\left(t_{2}-s\right)^{\alpha-1} \mathrm{~d} s-\int_{0}^{t_{1}}\left|\rho_{E}(s)\right|\left(t_{1}-s\right)^{\alpha-1} \mathrm{~d} s\right\}+\frac{r}{\Gamma(\alpha+1)}\left(t_{2}^{\alpha}-t_{1}^{\alpha}\right),
\end{aligned}
$$

and

$$
\begin{aligned}
& \left|D^{\alpha-1} K_{p}(I-Q) N u\left(t_{2}\right)-D^{\alpha-1} K_{p}(I-Q) N u\left(t_{1}\right)\right| \\
& \quad=\left|\int_{0}^{t_{2}}(I-Q) N u(s) \mathrm{d} s-\int_{0}^{t_{1}}(I-Q) N u(s) \mathrm{d} s\right| \\
& \quad \leq r\left(t_{2}-t_{1}\right)+\int_{t_{1}}^{t_{2}}\left|\rho_{E}(s)\right| \mathrm{d} s .
\end{aligned}
$$


Since $t^{\alpha}$ is uniformly continuous on $[0,1]$ and $\rho_{E}(t) \in L[0,1]$, so $K_{p}(I-Q) N(\bar{\Omega})$ and $D^{\alpha-1} K_{p}(I-Q) N(\bar{\Omega})$ are equicontinuous. By Lemma 2.8 , we get that $K_{p}(I-Q) N: Y \mapsto Y$ is completely continuous.

Lemma 3.6 Suppose (H1)-(H3) hold, then the set $\Omega_{1}=\{u \in \operatorname{dom} L \backslash \operatorname{Ker} L: L u=\lambda N u, \lambda \in$ $[0,1]\}$ is bounded.

Proof Taking any $u \in \Omega_{1}$, then we have $L u=\lambda N u$, which yields $\lambda \neq 0$ and $N u \in \operatorname{Im} L=$ $\operatorname{Ker} Q$, i.e., $Q N u=0$ for all $t \in[0,1]$. It follows from (H2) and (H3) that there exists $t_{0} \in[0,1]$ such that $\left|D_{0+}^{\alpha-1} u\left(t_{0}\right)\right|+\left|D_{0+}^{\alpha-2} u\left(t_{0}\right)\right| \leq A+B$. Then we can get that

$$
\begin{aligned}
& D_{0+}^{\alpha-1} u(t)=D_{0+}^{\alpha-1} u\left(t_{0}\right)+\int_{t_{0}}^{t} D_{0+}^{\alpha} u(s) \mathrm{d} s, \\
& D_{0+}^{\alpha-2} u(t)=D_{0+}^{\alpha-2} u\left(t_{0}\right)+\int_{t_{0}}^{t} D_{0+}^{\alpha-1} u(s) \mathrm{d} s .
\end{aligned}
$$

Furthermore, we have that, with setting $M=A+B$,

$$
\begin{aligned}
\left|D_{0+}^{\alpha-1} u(0)\right| & \leq\left\|D_{0+}^{\alpha-1} u(t)\right\|_{\infty} \leq\left|D_{0+}^{\alpha-1} u\left(t_{0}\right)\right|+\left\|D_{0+}^{\alpha} u\right\|_{1} \\
& \leq M+\|L u\|_{1} \leq M+\|N u\|_{1}, \\
\left|D_{0+}^{\alpha-2} u(0)\right| & \leq\left\|D_{0+}^{\alpha-2} u(t)\right\|_{\infty} \leq\left|D_{0+}^{\alpha-2} u\left(t_{0}\right)\right|+\left\|D_{0+}^{\alpha-1} u\right\|_{\infty} \\
& \leq\left|D_{0+}^{\alpha-2} u\left(t_{0}\right)\right|+\left|D_{0+}^{\alpha-1} u\left(t_{0}\right)\right|+\left\|D_{0+}^{\alpha} u\right\|_{1} \\
& \leq M+\|L u\|_{1} \leq M+\|N u\|_{1} .
\end{aligned}
$$

By (3.7)-(3.9) and Lemma 2.4, we have that

$$
\|P u\|_{Y} \leq \mu\left(M+\|N u\|_{1}\right) .
$$

As before, for any $u \in \Omega_{1}$, we have $(I-P)(u) \in \operatorname{dom} L \cap \operatorname{Ker} P$ and $L P(u)=0$. From Lemma 3.4 and for each $\lambda \in(0,1]$, we can get

$$
\|(I-P)(u)\|_{Y}=\left\|K_{p} L(I-P)(u)\right\|_{Y}=\left\|K_{p}(L u)\right\|_{Y} \leq \omega\|N u\|_{1} .
$$

Furthermore, we have

$$
\|u\|_{Y} \leq\|(I-P)(u)\|_{Y}+\|P(u)\|_{Y} \leq \rho\|N u\|_{1}+\mu M .
$$

By (H1) and the definition of $N$, we have

$$
\|u\|_{Y} \leq \rho\left[\|h\|_{1}\|u\|_{\infty}+\|r\|_{1}\left\|D_{0+}^{\alpha-1} u\right\|_{\infty}\|s\|_{1}\left\|D_{0+}^{\alpha-2} u\right\|_{\infty}+\|w\|_{1}\left\|D_{0+}^{\alpha-2} u\right\|_{\infty}^{\theta}+D\right]
$$

where $D=\|e\|_{1}+\mu M / \rho$. Since $\max \left\{\|u\|_{\infty},\left\|D_{0+}^{\alpha-1} u\right\|_{\infty},\left\|D_{0+}^{\alpha-2} u\right\|_{\infty}\right\} \leq\|u\|_{Y}$ and $\rho\left(\|h\|_{1}+\right.$ $\left.\|r\|_{1}+\|s\|_{1}\right)<1$ hold true, we can get that

$$
\|u\|_{\infty} \leq \frac{\rho}{1-\rho\|h\|_{1}}\left[\|r\|_{1}\left\|D_{0+}^{\alpha-1} u\right\|_{\infty}+\|s\|_{1}\left\|D_{0+}^{\alpha-2} u\right\|_{\infty}+\|w\|_{1}\left\|D_{0+}^{\alpha-2} u\right\|_{\infty}^{\theta}+D\right],
$$


which yield that

$$
\left\|D_{0+}^{\alpha-1} u\right\|_{\infty} \leq \frac{\rho}{1-\rho\|h\|_{1}-\rho\|r\|_{1}}\left[\|s\|_{1}\left\|D_{0+}^{\alpha-2} u\right\|_{\infty}+\|w\|_{1}\left\|D_{0+}^{\alpha-2} u\right\|_{\infty}^{\theta}+D\right] .
$$

Furthermore, from the previous inequalities, we know that

$$
\left\|D_{0+}^{\alpha-2} u\right\|_{\infty} \leq \frac{\rho}{1-\rho\|h\|_{1}-\rho\|r\|_{1}-\rho\|s\|_{1}}\left(\|w\|_{1}\left\|D_{0+}^{\alpha-2} u\right\|_{\infty}^{\theta}+D\right) .
$$

Since $\theta \in[0,1)$, there exist constants $m_{1}, m_{2}, m_{3}>0$ such that

$$
\left\|D_{0+}^{\alpha-2} u\right\|_{\infty} \leq m_{1}, \quad\left\|D_{0+}^{\alpha-1} u\right\|_{\infty} \leq m_{2}, \quad\|u\|_{\infty} \leq m_{3}
$$

Therefore, $\Omega_{1}$ is bounded.

Lemma 3.7 Suppose (H2) and (H3) hold, then the set $\Omega_{2}=\{u \in \operatorname{Ker} L: N u \in \operatorname{Im} L\}$ is bounded.

Proof For any $u \in \Omega_{2}$ and $a, b \in \mathbf{R}$, then $u(t)=a t^{\alpha-1}+b t^{\alpha-2}$ and $Q N u=0$. By (H2), we get that $\left|D_{0+}^{\alpha-1} u(t)\right|=|a \Gamma(\alpha)| \leq A$, then we have $|a| \leq A / \Gamma(\alpha)$. By (H3), we have that $\left|D_{0+}^{\alpha-2} u(t)\right|=|a t \Gamma(\alpha)+b \Gamma(\alpha-1)| \leq B$, thus $|b| \leq(B+A) / \Gamma(\alpha-1)$. Therefore, $\Omega_{2}$ is bounded.

Lemma 3.8 If the first parts of (H2) and (H3) hold, then the set $\Omega_{3}=\left\{u \in \operatorname{Ker} L: \lambda J^{-1} u+\right.$ $(1-\lambda) Q N u=0, \lambda \in[0,1]\}$ is bounded.

Proof Taking any $u \in \Omega_{3}$ and $a, b \in \mathbf{R}$, we have $u(t)=a t^{\alpha-1}+b t^{\alpha-2}$. For all $t \in[0,1]$, we define the isomorphism $J^{-1}: \operatorname{Ker} L \mapsto \operatorname{Im} Q$ by

$$
J^{-1}\left(a t^{\alpha-1}+b t^{\alpha-2}\right)=\frac{a^{2}}{\Lambda} t^{p-1}+\frac{b^{2}}{\Lambda} t^{q-2}
$$

By the definition of the set $\Omega_{3}$, we can get that

$$
\left\{\begin{array}{l}
\lambda a^{2}+(1-\lambda)\left[-\Lambda_{2} T_{1} N\left(a t^{\alpha-1}+b t^{\alpha-2}\right)+\Lambda_{4} T_{2} N\left(a t^{\alpha-1}+b t^{\alpha-2}\right)\right]=0 \\
\lambda b^{2}+(1-\lambda)\left[\Lambda_{1} T_{1} N\left(a t^{\alpha-1}+b t^{\alpha-2}\right)-\Lambda_{3} T_{2} N\left(a t^{\alpha-1}+b t^{\alpha-2}\right)\right]=0
\end{array}\right.
$$

If $\lambda=0$, we have

$$
T_{1} N\left(a t^{\alpha-1}+b t^{\alpha-2}\right)=0, \quad T_{2} N\left(a t^{\alpha-1}+b t^{\alpha-2}\right)=0 .
$$

By the first parts of (H2) and (H3), similar to the proof of Lemma 3.7, then

$$
|a| \leq \frac{A}{\Gamma(\alpha)}, \quad|b| \leq \frac{B+A}{\Gamma(\alpha-1)} .
$$

Therefore, $\Omega_{3}$ is bounded.

If $\lambda=1$, we have $a=b=0$. 
If $0<\lambda<1$, we get that $\left|D^{\alpha-1} u(t)\right| \leq A$ and $\left|D^{\alpha-2} u(t)\right| \leq B$, similar to the proof of Lemma 3.7, $\Omega_{3}$ is bounded. If else, we have that $\Lambda_{2} T_{1} N u-\Lambda_{4} T_{2} N u<0$ and $\Lambda_{1} T_{1} N u-$ $\Lambda_{3} T_{2} N u>0$. It contradicts (3.10), thus $\Omega_{3}$ is bounded.

Remark 3.9 If the other parts of $(\mathrm{H} 2)$ and (H3) hold, then the set $\Omega_{3}=\{u \in \operatorname{Ker} L$ : $\left.-\lambda J^{-1} u+(1-\lambda) Q N u=0, \lambda \in[0,1]\right\}$ is bounded.

Now with Lemmas 3.2-3.8 in hands, we can begin to prove our main result - Theorem 3.1.

Proof of Theorem 3.1 Assume that $\Omega$ is a bounded open set of $Y$ with $\bigcup_{i=1}^{3} \bar{\Omega} \subset \Omega$. By Lemma 3.5, $N$ is $L$-compact on $\bar{\Omega}$. Then by Lemmas 3.6 and 3.7, we have

(i) $L u \neq \lambda N u$ for every $(u, \lambda) \in[\operatorname{dom} L \backslash \operatorname{Ker} L \cap \partial \Omega] \times[0,1]$;

(ii) $N u \notin \operatorname{Im} L$ for every $u \in \operatorname{Ker} L \cap \partial \Omega$.

Finally, we will prove that (iii) of Lemma 2.6 is satisfied. We let $I$ as the identity operator in the Banach space $Y$ and $H(u, \lambda)= \pm \lambda J^{-1}(u)+(1-\lambda) Q N(u)$, according to Lemma 3.8 (or Remark 3.9) we know that for all $u \in \partial \Omega \cap \operatorname{Ker} L, H(u, \lambda) \neq 0$. By the homotopic property of degree, we have

$$
\begin{aligned}
\operatorname{deg}\left(\left.J Q N\right|_{\operatorname{Ker} L}, \operatorname{Ker} L \cap \Omega, 0\right) & =\operatorname{deg}(H(\cdot, 0), \operatorname{Ker} L \cap \Omega, 0) \\
& =\operatorname{deg}(H(\cdot, 1), \operatorname{Ker} L \cap \Omega, 0) \\
& =\operatorname{deg}( \pm I, \operatorname{Ker} L \cap \Omega, 0) \neq 0,
\end{aligned}
$$

so (iii) of Lemma 2.6 is satisfied.

Consequently, by Lemma 2.6, the equation $L u=N u$ has at least one solution in $\operatorname{dom} L \cap$ $\bar{\Omega}$. Namely, BVPs (1.1)-(1.2) have at least one solution in the space $Y$.

According to Theorem 3.1, we have the following corollary.

Corollary 3.10 Suppose that (H1) is replaced by the following condition,

(H4) there exist functions $h(t), r(t), s(t), w(t), e(t) \in L[0,1]$ and a constant $\theta \in[0,1)$ such that for all $(x, y, z) \in \mathbf{R}^{3}, t \in[0,1]$,

$$
|f(t, x, y, z)| \leq h(t)|x|+r(t)|y|+s(t)|z|+w(t)|y|^{\theta}+e(t)
$$

or

$$
|f(t, x, y, z)| \leq h(t)|x|+r(t)|y|+s(t)|z|+w(t)|x|^{\theta}+e(t)
$$

and the others in Theorem 3.1 are not changed, then BVPs (1.1)-(1.2) have at least one solution. 


\section{An example}

Example Consider the following boundary value problem for all $t \in(0,1)$ :

$$
\left\{\begin{array}{l}
D^{\frac{13}{5}} u(t)=\frac{7 u(t)}{200}+\frac{9 D_{0+}^{\frac{8}{5}} u(t)}{500}+\frac{D_{0+}^{\frac{3}{5}} u(t)}{60}+\frac{3 \sin ^{\frac{1}{7}} u(t)}{80}+\cos ^{2} t+1, \\
I_{0+}^{\frac{2}{5}} u(0)=0, \quad D_{0+}^{\frac{8}{5}} u(1)=\frac{2}{5} D_{0+}^{\frac{8}{5}} u\left(\frac{1}{3}\right)+\frac{3}{5} D_{0+}^{\frac{8}{5}} u\left(\frac{1}{2}\right), \\
D_{0+}^{\frac{3}{5}} u(1)=-6 D_{0+}^{\frac{3}{5}} u\left(\frac{1}{8}\right)+7 D_{0+}^{\frac{3}{5}} u\left(\frac{1}{4}\right) .
\end{array}\right.
$$

Let $\alpha=13 / 5$, and $a_{1}=2 / 5, \xi_{1}=1 / 3, a_{2}=3 / 5, \xi_{2}=1 / 2, b_{1}=-6, \eta_{1}=1 / 8, b_{2}=7, \eta_{2}=$ $1 / 4$. We can get that the condition (C) holds, i.e., $\sum_{i=1}^{2} a_{i}=1, \sum_{i=1}^{2} b_{i} \eta_{i}=1, \sum_{i=1}^{2} b_{i}=1$. Moreover,

$$
f(t, x, y, z)=\frac{7}{200} x+\frac{9}{500} y+\frac{1}{60} z+\frac{3}{80} \sin ^{1 / 7} x+\cos ^{2} t+1 .
$$

Thus, we have

$$
|f(t, x, y, z)| \leq \frac{7}{200}|x|+\frac{9}{500}|y|+\frac{1}{60}|z|+\frac{3}{80}|x|^{1 / 7}+2 .
$$

Taking $h=7 / 200, r=9 / 500, s=1 / 60, w=3 / 80, e=2, \Lambda_{1}=\left(1-\sum_{i=1}^{2} b_{i} \eta_{i}^{2}\right) / 2, \Lambda_{2}=$ $\left(1-\sum_{i=1}^{2} b_{i} \eta_{i}^{2}\right) / 2, \Lambda_{3}=1-\sum_{i=1}^{2} a_{i} \xi_{i}, \Lambda_{4}=1-\sum_{i=1}^{2} a_{i} \xi_{i}, \mu=3+\Gamma^{-1}(13 / 5)+\Gamma^{-1}(8 / 5)$, $\omega=2+\Gamma^{-1}(13 / 5), \rho=5+2 \Gamma^{-1}(13 / 5)+\Gamma^{-1}(8 / 5)$, we can calculate that (H1)-(H3) hold. Furthermore, we can get

$$
\rho\left(\|h\|_{1}+\|r\|_{1}+\|s\|_{1}\right)=0.52754
$$

By Corollary 3.10, the BVP (4.1) has at least one solution in $C^{8 / 5}[0,1]$.

\section{Competing interests}

The authors declare that they have no competing interests.

\section{Author's contributions}

NX designed all the steps of proof in this research and also wrote the article. WBL suggested many good ideas in this article. LSX helped to draft the first manuscript and gave an example to illustrate our result. All authors read and approved the final manuscript.

\section{Acknowledgement}

The authors would like to acknowledge the anonymous referee for many helpful comments and valuable suggestions on this article. This work is sponsored by Fundamental Research Funds for the Central Universities (2012LWB44).

\section{Received: 17 January 2012 Accepted: 18 May 2012 Published: 28 June 2012}

\section{References}

1. Sabatier, J, Agrawal, O, Machado, J: Advances in Fractional Calculus: Theoretical Developments and Applications in Physics and Engineering. Springer, Berlin (2007)

2. Samko, S, Kilbas, A, Marichev, O: Fractional Integrals and Derivatives: Theory and Applications. Gordon \& Breach, New York (1993)

3. Kilbas, A, Srivastava, H, Trujillo, J: Theory and Applications of Fractional Differential Equations. Elsevier, Amsterdam (2006)

4. Momani, S, Odibat, Z: Numerical comparison of methods for solving linear differential equations of fractional order. Chaos Solitons Fractals 31(5), 1248-1255 (2007)

5. Bai, Z, Lü, H: Positive solutions for boundary value problem of nonlinear fractional differential equation. J. Math. Anal. Appl. 311(2), 495-505 (2005)

6. $\mathrm{Xu}, \mathrm{X}$, Jiang, $\mathrm{D}$, Yuan, $\mathrm{C}$ : Multiple positive solutions for the boundary value problem of a nonlinear fractional differential equation. Nonlinear Anal. 71(10), 4676-4688 (2009) 
7. Kaufmann, E, Mboumi, E: Positive solutions of a boundary value problem for a nonlinear fractional differential equation. Electron. J. Qual. Theory Differ. Equ. 3, 1-11 (2008)

8. Zhang, S: Positive solutions for boundary-value problems of nonlinear fractional differential equations. Electron. J. Differ. Equ. 2006(36), 1-12 (2006)

9. Benchohra, M, Hamani, S, Ntouyas, S: Boundary value problems for differential equations with fractional order and nonlocal conditions. Nonlinear Anal. 71(7-8), 2391-2396 (2009)

10. Liang, S, Zhang, J: Positive solutions for boundary value problems of nonlinear fractional differential equation. Nonlinear Anal. 71(11), 5545-5550 (2009)

11. El-Sayed, A: Nonlinear functional differential equations of arbitrary orders. Nonlinear Anal. 33(2), 181-186 (1998)

12. Agarwal, R, Lakshmikantham, $V$, Nieto, J: On the concept of solution for fractional differential equations with uncertainty. Nonlinear Anal. 72(6), 2859-2862 (2010)

13. Chang, Y, Nieto, J: Some new existence results for fractional differential inclusions with boundary conditions. Math. Comput. Model. 49(3-4), 605-609 (2009)

14. Zhang, Y, Bai, Z, Feng, T: Existence results for a coupled system of nonlinear fractional three-point boundary value problems at resonance. Comput. Math. Appl. 61(4), 1032-1047 (2011)

15. Kosmatov, N: A boundary value problem of fractional order at resonance. Electron. J. Differ. Equ. 135, 1-10 (2010)

16. Zhang, Y, Bai, Z: Existence of solutions for nonlinear fractional three-point boundary value problems at resonance. J. Appl. Math. Comput. 36, 417-440 (2011)

17. Bai, Z: On solutions of some fractional $m$-point boundary value problems at resonance. Electron. J. Qual. Theory Differ. Equ. 37, 1-15 (2010)

18. Bai, Z, Zhang, Y: The existence of solutions for a fractional multi-point boundary value problem. Comput. Math. Appl. 60(8), 2364-2372 (2010)

19. Jiang, W: The existence of solutions to boundary value problems of fractional differential equations at resonance. Nonlinear Anal. 74(5), 1987-1994 (2011)

20. Mawhin, J: Topological degree and boundary value problems for nonlinear differential equations. In: Furi, M, Zecca, P (eds.) Topological Methods for Ordinary Differential Equations. Lecture Notes in Mathematics, vol. 1537, pp. 74-142. Springer, Berlin (1993)

doi:10.1186/1687-2770-2012-65

Cite this article as: $\mathrm{Xu}$ et al.: The existence of solutions for nonlinear fractional multipoint boundary value problems at resonance. Boundary Value Problems 2012 2012:65.

\section{Submit your manuscript to a SpringerOpen ${ }^{\circ}$ journal and benefit from:}

- Convenient online submission

Rigorous peer review

- Immediate publication on acceptance

- Open access: articles freely available online

- High visibility within the field

- Retaining the copyright to your article 